\title{
SOME UNIQUENESS THEOREMS FOR FUNGTIONAL EQUATIONS
}

\author{
T. D. HOWROYD
}

(Received 23 June 1967)

The generalized Pexider equation

$$
g(F(x, y))=H(f(x), f(y), x, y)
$$

where $f$ and $g$ are unknown and $x, y$, are real, has been discussed by J. Aczél [1] and J. Aczél and M. Hosszú [2]. In [2] it is shown that if $F$ is continuous and $F$ and $H$ are strictly increasing in their first variables and strictly decreasing in their second variables, then two initial conditions suffice to determine at most one continuous solution $f$ of $(1)$. We extend these results to strictly increasing and strictly decreasing functions $F$ and derive results for strictly monotonic $F$ and $H$.

As in [2] we call $F$ reflexive at $a$ if $F(a, a)=a$, i.e. if $x=a$ is a fixedpoint of $F(x, x)$. We sometimes omit parentheses where the meaning is clear, e.g. $f x=f(x)$. We use the standard notation for iterates, e.g. $\alpha^{0} x=x$, $\alpha^{n+1} x=\alpha \alpha^{n} x, n=0,1, \cdots$.

THEOREM 1. Let $I$ be an interval. Let $F$ be continuous and strictly increasing (or decreasing) in $I \times I$. Let $N$ be a Hausdorff space. Let $H$ be defined in $N \times N \times I \times I$ such that

$$
H\left(u_{1}, u_{1}, x, x\right)=H\left(u_{2}, u_{2}, x, x\right) \text { implies } u_{1}=u_{2} ;
$$

and either

$$
H\left(u, u_{1}, x, y\right)=H\left(u, u_{2}, x, y\right) \text { implies } u_{1}=u_{2},
$$

or

$$
H\left(u_{1}, u, x, y\right)=H\left(u_{2}, u, x, y\right) \text { implies } u_{1}=u_{2} .
$$

Let $g=g_{1}$ and $f=f_{1}$, also $g=g_{2}$ and $f=f_{2}$, satisfy (1) in $I \times I$. Let $f_{1}$ and $f_{2}$ be continuous maps of $I$ into $N$, and $a, b \in I$ such that $a \neq b$, $f_{1}(a)=f_{2}(a), f_{1}(b)=f_{2}(b)$. Then $f_{1}=f_{2}$ in $I$ and $g_{1}=g_{2}$ in the range of $F$ on $I \times I$.

Let $g=f$ in (1). If $F$ is reflexive everywhere in $I$ the condition (I) is redundant. If $F(a, a) \in I$ and $F$ is not reflexive at a the condition $f_{1}(b)=f_{2}(b)$ 
is redundant. If $I$ is the interval $0 \leqq x<d$ and $F(x, y)=x+y$ the conditions (II) and (III) are redundant; and provided $a>0$ the condition $f_{1}(b)=f_{2}(b)$ is redundant.

Proof. Let $p x=F(x, x), x \in I$. Then $p$ is continuous and strictly monotonic and has a unique inverse $p^{-1}$. We may define $G=p^{-1} F$. Then $G$ has domain $I \times I$ and range $I$, and is continuous, strictly increasing in both variables, and reflexive in $I$.

Let (II) hold. We may suppose $a<b$. Let $\alpha x=G(a, x), \beta x=G(b, x)$, $x \in I$. Then $\alpha$ and $\beta$ are continuous, strictly increasing, and have unique inverses. Also $\alpha a=a, \beta b=b, \alpha x<x$ for $x>a, \beta x>x$ for $x<b$.

We may substitute $G(x, y)$ for $x$ and $y$ in (1), then $f_{1}$ and $f_{2}$ satisfy the functional equation

$$
H(f x, f y, x, y)=H(f G(x, y), f G(x, y), G(x, y), G(x, y))
$$

in $I \times I$. Substitute $x=a$ and $y=b, \alpha b, \alpha^{2} b, \cdots$, successively in (2), then (I) implies $f_{1} \alpha^{n} b=f_{2} \alpha^{n} b(n=1,2, \cdots)$.

Let $I^{\prime}$ be the interval $a \leqq x \leqq b$. Assume that $f_{1} \neq f_{2}$ in $I^{\prime}$. Then it is not true that $f_{1}=f_{2}$ in a set which is dense in $I^{\prime}$. Hence there exist $a_{1}, b_{1} \in I^{\prime}$ such that $f_{1}$ and $f_{2}$ intersect at $a_{1}$ and $b_{1}$ but are different everywhere in $a_{1}<x<b_{1}$. Let $c_{n}=G\left(\alpha^{n} b, a_{1}\right)$. Then (I) and (2) imply $f_{1} c_{n}=f_{2} c_{n}$. But $\alpha^{n} b \rightarrow a, c_{n} \rightarrow \alpha a_{1}$, and since these sequences strictly decrease, there exists an integer $m$ such that

$$
\alpha a_{1}<c_{m}<\alpha b_{1}, a_{1}<\alpha^{-1} c_{m}<b_{1} .
$$

But (II) and (2) with $x=a$ and $y=\alpha^{-1} c_{m}$ imply $f_{1} \alpha^{-1} c_{m}=f_{2} \alpha^{-1} c_{m}$, which contradicts our assumption. Hence $f_{1}=f_{2}$ in $I^{\prime}$.

If $x \in I, x \geqq a$, then $\alpha^{a} x \in I^{\prime}$ for some positive integer $q$; hence $f_{1} \alpha^{q} x=f_{2} \alpha^{q} x$, and (II) and (2) with $x=a$ and $\left\langle y=\alpha^{q-1} x, \alpha^{q-2} x, \cdots, x,\right\rangle$ successively, imply $f_{1} x=f_{2} x$. If $x \in I, x \leqq b$, then $\beta^{r} x \in I^{\prime}$ for some positive integer $r$; hence $f_{1} \beta^{r} x=f_{2} \beta^{r} x$, and (II) and (2) with $x=b$ and

$$
y=\beta^{r-1} x, \beta^{r-2} x, \cdots, x,
$$

successively, imply $f_{1} x=f_{2} x$. Hence $f_{1}=f_{2}$ in $I$.

If instead of (II), (III) holds, the above process may be repeated with $G(a, x)$ and $G(b, x)$ replaced by $G(x, a)$ and $G(x, b)$, respectively.

Let $g=f$ in (1). If $F(x, x) \equiv x, x \in I$, then $f_{1}$ and $f_{2}$ satisfy the equation $f x=H(f x, f x, x, x)$ in $I$, which is sufficient for the proof of the theorem, instead of (I). If $F(a, a) \in I$ and $F(a, a) \neq a$ then $f_{1} a=f_{2} a$ implies $f_{1} F(a, a)=f_{2} F(a, a)$, and we may take $b=F(a, a)$.

Let $g=f$ in (1), $I$ be the interval $0 \leqq x<d$, and $F(x, y)=x+y$. If $t \in I$ such that $f_{1} t=f_{2} t$ then $f_{k} t=H\left(f_{k} \frac{1}{2} t, f_{k} \frac{1}{2} t, \frac{1}{2} t, \frac{1}{2} t\right)(k=1,2)$, and (I) implies $f_{1} \frac{1}{2} t=t_{2} \frac{1}{2} t$. If also $n t \in I$ for some positive integer $n$, then 


$$
\begin{aligned}
& f_{1} 2 t=H\left(f_{1} t, f_{1} t, t, t\right)=f_{2} 2 t, \\
& f_{1} 3 t=H\left(f_{1} 2 t, f_{1} t, 2 t, t\right)=f_{2} 3 t,
\end{aligned}
$$

and by induction $f_{1} n t=f_{2} n t$. Now, either $a>0$ or $b>0$, and if $a>0$ the points $m 2^{-n} a$ ( $m$ and $n$ positive integers) are dense in $I$. But $f_{1} a=f_{2} a$ implies $f_{1} m 2^{-n} a=f_{2} m 2^{-n} a$, hence $f_{1}=f_{2}$ in $I$.

THEOREM 2. Let $g=f$ in (1). Let $F$ be reflexive at $a$, continuous and strictly monotonic in each variable in a neighbourhood of $(a, a)$. For $(x, y)$ in a neighbourhood of $(a, a)$ and $(u, v)$ in a neighbourhood of $(c, c)$ let $H(u, v, x, y)$ be strictly monotonic in $u$ and $v$. Let $f_{1}$ and $f_{2}$ be continuous solutions of (1) in a neighbourhood of $(a, a)$, and $f_{1}(a)=f_{2}(a)=c$. Then there exists a neighbourhood of $a$ in which either $f_{1}$ and $f_{2}$ have only the one point in common or are identical.

Proor. $f_{1}$ and $f_{2}$ will satisfy

$$
f E(x, y)=J(f x, f y, x, y)
$$

for $(x, y)$ in a neighbourhood of $(a, a)$, where

$$
E(x, y)=F\{F(x, a), F(a, y)\}
$$

and

$$
J(u, v, x, y)=H\{H(u, c, x, a), H(c, v, a, y), F(x, a), F(a, y)\} .
$$

But $E(a, a)=a$ and $E$ is continuous and strictly increasing in a neighbourhood of $(a, a)$. Also for $(x, y)$ in a neighbourhood of $(a, a)$ and $(u, v)$ in a neighbourhood of $(c, c), J(u, v, x, y)$ is strictly increasing in $u$ and $v$. Hence there exists a neighbourhood $N$ of $c$ and a neighbourhood $I$ of $a$ such that $f_{1}$ and $f_{2}, E$ and $J$ satisfy the hypotheses of Theorem 1 . Then $I$ is the required neighbourhood of $a$.

Example. The equation

$$
f(\sqrt{|x y|})=\sqrt{f(x) f(y)}
$$

illustrates both theorems. The function $F(x, y)=\sqrt{|x y|}$ is not strictly monotonic in either variable in any region including the origin; indeed there is an infinite number of continuous solutions of (4) of the form $A|x|^{B}$ which pass through the origin and any other point with positive $y$ coordinate. If we consider (4) only for negative $x$ and $y$ we may apply Theorem 1 . However although $F$ is then not reflexive anywhere both initial conditions are necessary; in this case the condition $F(a, a) \in I$ in Theorem 1 is not fulfilled for any $a \in I$. 
NotE. The reflexive case in Theorem 1 is already contained in [1] as a special case.

\section{References}

[1] J. Aczél, 'Ein Eindeutigkeitssatz in der Theorie der Funktional-gleichungen und einige ihrer Anwendungen', Acta Math. Acad. Sci. Hung. 15 (1964), 355-362.

[2] J. Aczél and M. Hosszú, 'Further Uniqueness Theorems for Functional Equations', Acta Math. Acad. Sci. Hung. 16 (1965), 51-55.

University of Melbourne 